Volume $10 \mid 2012$

Habiter : l'ancrage territorial comme support d'éducation à l'environnemental

\title{
Les territoires des Agendas 21 scolaires
}

Dominique Prost

\section{(2) OpenEdition \\ Journals}

Édition électronique

URL : http://journals.openedition.org/ere/1203

DOI : $10.4000 /$ ere. 1203

ISSN : 2561-2271

Éditeur

Centr'ERE

Référence électronique

Dominique Prost, "Les territoires des Agendas 21 scolaires », Éducation relative à l'environnement [En ligne], Volume 10 | 2012, mis en ligne le 20 décembre 2012, consulté le 21 février 2020. URL : http:// journals.openedition.org/ere/1203; DOI : 10.4000/ere.1203 


\title{
Les territoires des Agendas 21 scolaires
}

\author{
Dominique Prost
}

1 L'institutionnalisation de l'éducation au développement durable (EDD) impulsée d'en haut par le ministère de l'Éducation nationale est récente. C'est en 2007 que sont lancées les démarches de type Agenda 21 scolaire, sous le nom d'établissement en démarche de développement durable (E3D). Plus d'un millier d'établissements scolaires sont actuellement concernés (Musset, 2010) et divers labels se juxtaposent ${ }^{1}$ sans qu'il soit toujours possible de voir une réelle différence dans les contenus ou les pratiques. Toutes ces démarches se réclament d'un « ancrage territorial ». Comment l'institution scolaire et les acteurs de terrain abordent-ils la question du territoire? Quelles représentations en ont-ils? Quelles actions mettent-ils en œuvre pour s'y ancrer?

2 Pour aborder cette question, il est essentiel d'analyser le point de vue institutionnel exprimé dans les textes officiels et de s'interroger sur les cadres théoriques explicites ou implicites qui sous-tendent les références au territoire. Par ailleurs il faut mettre en regard ces injonctions venues d'en haut avec la réalité des pratiques développées sur le terrain. Une enquête sur les Agendas 21 scolaires en Aquitaine a été réalisée en 2009 auprès des divers partenaires extérieurs associés à la démarche (collectivités territoriales, Rectorat, associations). Ceci a permis de sélectionner un échantillon de dix lycées et collèges pour analyser la démarche adoptée, le contenu des actions et rencontrer les porteurs du projet. Dans un deuxième temps une analyse rapide des expériences menées en Rhône Alpes et en Midi Pyrénées² a permis une approche comparative à l'échelle régionale, replacée dans un contexte national élargi grâce à une rencontre avec le Comité 21.

\section{La dimension territoriale des Agendas 21}

3 Deux circulaires ministérielles (ministère de l'Éducation nationale, 2004, 2007) définissent ce que l'institution scolaire entend par Éducation au développement durable (EDD). On trouve une seule occurrence du terme territoire dans la circulaire 
ministérielle de 2004 : l'EDD « intègre pleinement par le regard porté au territoire les valeurs associées à un développement solidaire " (ministère de l'Éducation nationale, 2004). Par contre en 2007, un long développement est consacré au territoire :

Le travail dans les disciplines peut s'appuyer sur des exemples, des situations, des études de cas, des problématiques identifiées dans le territoire de l'établissement, à différentes échelles... Ainsi la construction des contenus, des concepts précisés dans les programmes nationaux pourra se faire en mettant en parallèle une meilleure compréhension du territoire, de ses enjeux, et des caractéristiques de son développement... Cette démarche permettra en outre de comprendre la complexité des situations, de former à l'exercice local de la citoyenneté: la proximité favorisant l'intérêt des élèves et les possibilités d'initiatives concrètes (ministère de l'Éducation nationale, 2007).

4 Ces deux textes proposent différentes approches du territoire :

- Le territoire abordé comme objet de connaissance permet d'acquérir des savoirs pour en comprendre la complexité ;

- Le territoire est aussi vu comme terrain d'expérimentation dans le cadre d'une approche pragmatique centrée sur la résolution des problèmes ;

- Le territoire doit permettre l'apprentissage des valeurs de solidarité et de citoyenneté ;

- Le territoire est enfin un outil méthodologique permettant d'articuler le local au global, d'ouvrir l'école sur le monde à partir de territoires de proximité définis dans leur cadre administratif qui est celui de la gestion des établissements.

5 Cette vision du territoire renvoie à divers cadres de référence qui relèvent principalement des domaines économiques et sociaux.

- L'entrée économique, souvent privilégiée, considère le territoire comme la base matérielle de la vie sociale et une ressource pour les sociétés. Elle renvoie à des approches gestionnaires qui se veulent éco-responsables;

- L'Agenda 21 questionne le modèle de développement sans aller jusqu'à une réelle approche critique qui ferait référence aux théories de l'écodéveloppement ou à la décroissance. Il s'agit d'une conception relativement floue: «Le développement solidaire, à toutes les échelles d'espace, constitue un concept associé au développement durable » (Bonhoure et Faucqueur, 2004, p. 67);

- Certains voient l'ancrage territorial comme «l'ensemble des connaissances pluridisciplinaires nécessaires pour connaître et agir sur des territoires... en un savoir situé, local, particulier ", il s'agit de mobiliser des savoirs locaux et de s'inscrire dans "un processus de patrimonialisation du territoire » (Legardez, 2008, p. 5);

- Pour les sciences sociales, et plus particulièrement pour la géographie, le territoire est défini comme un espace approprié, « lieu d'une expérience individuelle et collective constitutive des identités, où se construit la relation de soi au monde » (Levy et Lussault, 2003, p. 912).

\section{La diversité des représentations du territoire dans les pratiques d'Agendas 21 scolaires}

6 L'enquête réalisée en Aquitaine révèle que les thèmes abordés s'inscrivent dans des territorialités variables selon les acteurs, la communauté éducative reste souvent centrée sur l'établissement scolaire alors que leurs partenaires apportent une ouverture sur le monde extérieur. 


\section{Le point de vue des acteurs de l'institution scolaire}

7 Pour ceux-ci, l'établissement scolaire, souvent vécu par ses usagers comme simple lieu de passage temporaire, est au centre du projet : «L'établissement est l'espace privilégié de l'éducation au développement durable dont il devient le premier lieu et objet d'application " (Bregeon, Faucheux et Rochet, 2008, p. 6). Des actions matérielles et symboliques doivent permettre de changer le rapport au lieu pour en faire un lieu de vie et construire un vivre ensemble.

a. Un ancrage matériel: les Agendas 21 scolaires développent de nombreux projets pour une gestion éco-responsable des établissements, souvent sectoriels ils concernent en priorité le bâti et la gestion des flux. Ces actions ont l'avantage de mettre en relation élèves, enseignants, personnels de services avec des partenaires extérieurs. Elles permettent aussi d'obtenir des résultats rapidement quantifiables.

Il s'agit d'une approche pragmatique portée le plus souvent par les gestionnaires. Souvent prescriptive, elle vise à résoudre les problèmes et à changer les comportements par une éducation aux bonnes pratiques (Musset, 2010), parfois manipulatrice dans la mesure où, par le biais des enfants, ces bonnes pratiques se diffuseraient auprès des parents (Bachelart, 2006). Cette approche économiciste et techno-scientifique du développement durable risque de se limiter à une vision « ressourciste et gestionnaire du monde » (Sauvé, 2007), sans réelle réflexion critique sur les controverses, les incertitudes, ou les systèmes de valeurs en présence.

a. Un ancrage symbolique: s'approprier l'espace c'est aussi marquer son territoire. Divers aménagements permettent de transformer les lieux : jardins scolaires, aménagements de plans d'eau, plantations d'arbres s'inscrivent dans le territoire par le choix d'essences locales et par une réflexion sur les écosystèmes. Les établissements agricoles sont des précurseurs en ce domaine grâce à leurs terrains d'expérimentation.

b. Construire un "vivre ensemble » : la démarche participative et de nouveaux modes de prise de décision transforment en profondeur le fonctionnement des établissements pour passer d'initiatives impulsées d'en haut à des projets émanant de la base. La mise en œuvre mobilise une équipe qui associe des personnels habituellement peu impliqués dans les démarches éducatives. Dans quelques cas, on peut parler d'une nouvelle gouvernance qui permet de passer d'un système éducatif fondé traditionnellement sur une structure verticale et cloisonnée à une communauté scolaire soudée autour de projets communs.

c. Une ouverture prudente sur les territoires de proximité ? «Il ne s'agit plus de penser un projet dans le cadre fermé de l'établissement, répondant aux préoccupations internes des personnels, mais de confronter ces préoccupations à celles des acteurs du territoire » (Peltier, 2010, p. 3).

d. L'ouverture sur l'extérieur passe par des partenariats avec les collectivités territoriales et les associations ce qui permet de confronter des représentations du territoire différentes et de décentrer les projets.

\section{Les territoires des partenaires extérieurs}

a. L'approche des collectivités territoriales: elles sont souvent à l'initiative des Agendas 21 scolaires auxquels elles apportent un soutien technique et financier. Les projets s'inscrivent dans leur territoire de légitimité politique et dans leurs priorités du moment, avec des préoccupations de gestionnaires, puisqu'elles ont en charge, selon leur niveau de compétences, écoles, collèges et lycées. 
En Aquitaine la région, le département de la Gironde et la Communauté urbaine de Bordeaux (CUB) ont signé une convention pour harmoniser leurs pratiques concernant les Agendas 21 scolaires : sélection tous les ans d'un nombre limité d'établissements après appel d'offres, aide de 3000 euros sur trois ans pour financer un accompagnement associatif.

L'objectif premier des collectivités est d'intervenir sur le bâti en vue d'une gestion plus économe, de privilégier des thèmes qui s'inscrivent dans leurs propres projets (plan de développement durable de la CUB, Agenda 21 de la Gironde, plan climat régional), et de valoriser ces actions à l'occasion d'événements médiatisés ou sur leurs sites Internet.

Tableau 1 : Les thèmes proposés par les collectivités territoriales en Aquitaine

\begin{tabular}{|c|c|c|}
\hline CUB (15 écoles) & Gironde (17 collèges) & Aquitaine (19 lycées) \\
\hline $\begin{array}{l}\text {-Éco-mobilité, } \\
\text { - Éco-consommation, } \\
\text { - Éco-construction, } \\
\text { - Écosystème, } \\
\text { - Écocitoyenneté. }\end{array}$ & $\begin{array}{l}3 \text { thèmes obligatoires: } \\
\text { - Consommation responsable, } \\
\text { - Respect de l'environnement, } \\
\text { - Écocitoyenneté. } \\
\text { 2 thèmes au choix: } \\
\text { - Lutte contre le changement climatique, } \\
\text { - Satisfaction des besoins fondamentaux, } \\
\text { - Développement équilibré, solidaire et } \\
\text { responsable du territoire girondin, } \\
\text { - Solidarité entre les territoires et les } \\
\text { générations, } \\
\text { - Préservation et valorisation des ressources } \\
\text { naturelles, } \\
\text { - Dynamique citoyenne et sens de l'action } \\
\text { collective, } \\
\text { - Innovation et changement des pratiques. }\end{array}$ & $\begin{array}{l}\text { - Lutte contre le changement climatique, } \\
\text { - Renouveau des liens avec la nature et la } \\
\text { biodiversité, } \\
\text { - Lutte contre les inégalités, } \\
\text { - Solidarités avec les populations les plus } \\
\text { démunies ici et dans le monde. }\end{array}$ \\
\hline
\end{tabular}

a. L'approche des associations : les champs de compétences et les pratiques des associations partenaires étant très variés, celles-ci ont des approches différentes du territoire. Cependant, toutes apportent une expérience et une ouverture sur le monde extérieur par des rencontres avec des acteurs de terrain et un fonctionnement en réseau (cf. Graine Aquitaine, Ifrée en Poitou-Charentes, CIRCEE en Rhône-Alpes, etc.). Cette mise en réseau modifie la manière de travailler et permet le partage de ressources et les échanges d'expériences dans un cadre territorial élargi, national et international.

b. Habiter un territoire élargi : dans certains cas un "vivre ensemble élargi » se construit à travers des actions concrètes qui ouvrent l'établissement sur une communauté de vie à l'échelle du quartier, de la commune ou de la ville. Des liens de voisinage et d'échange se tissent. L'ancrage territorial se révèle alors « un outil possible pour modifier les rapports sociaux » (Fournier, 2007, p. 34).

\section{Les limites des Agendas 21 scolaires}

8 La diversité des représentations du territoire et la multiplicité des acteurs sont source de contradictions. Par ailleurs, la durabilité des projets et de l'ancrage territorial restent fragiles.

a. Des territoires à géométrie variable qui ne se recoupent pas : les territoires d'appartenance de chacun sont multiples et mobiles, des contradictions existent entre les démarches centrées sur l'établissement et l'ouverture sur les territoires. 
b. Comment passer des territoires de proximité à une vision globale et planétaire? Les approches à l'échelle globale restent exceptionnelles (à l'exception de thématiques du type plan climat ou actions de solidarité nord-sud). Bien souvent les Agendas restent à l'échelle locale.

c. Quelle durabilité des projets, des équipes et des territoires? Certains distinguent une durabilité faible, où se situent la majorité des actions, et une durabilité forte remettant en cause le fonctionnement institutionnel et social.

On constate sur le terrain de fortes résistances face à la démarche participative qui remet en cause un système hiérarchique et cloisonné fondé sur le découpage en disciplines scolaires. Par ailleurs, les projets reposent sur un réseau relationnel fondé sur des liens personnels. Or, élèves et personnels sont mobiles, de plus les expériences en cours reposent sur le bénévolat, ce qui présente un risque certain d'usure. Enfin, l'accompagnement associatif financé par les collectivités est limité dans le temps et n'est pas extensible à l'ensemble des établissements scolaires, car trop coûteux. Comment les retours d'expériences vont-ils être diffusés et appropriés par d'autres établissements scolaires?

\section{Conclusion}

Malgré ces interrogations et ces difficultés, les Agendas 21 scolaires ont l'ambition de dépasser les approches disciplinaires ou sectorielles pour construire des projets d'établissements globaux et cohérents, abordant des questions complexes et ouvrant l'école sur le monde. Les partenariats construits dans l'action permettent cette ouverture sur des territoires et des réseaux sociaux de proximité porteurs d'un vivre ensemble. Habitant quelque part, ancrés dans des territoires de proximité porteurs de mémoire et d'identité, il devient sans doute possible pour les élèves de s'ouvrir sur un ailleurs, de se décentrer pour saisir les enjeux planétaires et habiter la terre de manière plus consciente et solidaire.

\section{BIBLIOGRAPHIE}

Bachelart, D. (2006). Le développement durable bouscule l'éducation à l'environnement. Territoires, 466, 8-11.

Bonhoure, G. et Faucqueur, C. (2004). L'éducation à l'environnement pour un développement durable dans les établissements scolaires. In Ricard, M., Colloque international sur l'éducation à l'environnement pour un développement durable (p. 63-75). Paris : ministère de l'Éducation nationale. Bregeon, J., Faucheux, S. et Rochet, C. (2008). Rapport du groupe de travail interministériel sur l'éducation au développement durable. Paris : ministère de l'Environnement.

Comite 21 (2006). De l'école au campus, agir ensemble pour le développement durable. Guide méthodologique de l'Agenda 21 scolaire. Paris : Sceren, CRDP. 
Fournier, J.-M. (2007). Géographie sociale et territoires. De la confusion sémantique à l'utilité sociale? ESO, 28, 29-35.

Legardez, A. (2008). Co-construire des savoirs critiques sur l'environnement; un contre-feu au nouveau catéchisme du développement durable ? Communication présentée lors du colloque « Éthique et éducation à l'environnement ", Ifrée, La Rochelle, juin 2008.

Levy, J. et Lussault, M. (2003). Dictionnaire de la géographie et de l'espace des sociétés. Paris : Belin. Ministère de l'Éducation nationale (2004). Généralisation d'une éducation à l'environnement pour un développement durable - rentrée 2004, Bulletin officiel de l'Éducation nationale, $\mathrm{n}^{\circ} 28,15$ juillet 2004, Circulaire $n^{\circ} 2004-110$ du 8 juillet 2004.

Ministère de l'Éducation nationale (2004). Seconde phase de généralisation de l'éducation au développement durable (E.D.D.), Bulletin officiel de l'Éducation nationale, n 14, 5 avril 2007, Circulaire $n^{\circ}$ 2007-077 du 29 mars 2007.

Musset, M. (2010). L'éducation au développement durable. Dossier d'actualité $n^{\circ} 56$. Paris : INRP.

Peltier, C. (2010). L'Agenda 21 : outil stratégique de généralisation de l'EDD pour les établissements de l'enseignement agricole. Cahiers pédagogiques, 478. Consulté le 7 mai 2012 sur http://www.cahiers-pedagogiques.com/L-Agenda-21-outil-strategique-de.html

Sauvé, L. (2007). L'équivoque du développement durable. Chemin de Traverse, 4, 31-47

\section{NOTES}

1. Éco-écoles, éco-responsables, E3D, Agendas 21 scolaires, etc.

2. Deux sources ont été utilisées : un rapport réalisé pour le Conseil Régional Rhône-Alpes par l'association Les amis de Circée sur les établissements éco-responsables, en octobre 2009 (Recensement des établissements éco-responsables de la région Rhone-Alpes, http:// lewebpedagogique.com/comite21-agenda21scolaire/recensement-des-etablissements-ecoresponsables-de-la-region-rhone-alpes/); un sondage sur une vingtaine de sites Internet consacrés aux Agendas 21 scolaires de lycées et collèges situés dans les régions Rhône Alpes et Midi-Pyrénées.

\section{AUTEUR \\ DOMINIQUE PROST}

Maître de conférences de géographie à l'IUFM d'Aquitaine et membre de l'UMR $5185 \mathrm{du}$ laboratoire ADES (Aménagement Développement Environnement Santé et Société) au CNRSUniversité de Bordeaux. Ses travaux de recherche portent sur l'éducation au développement durable au sein de l'IUFM, sur la géographie sociale et l'aménagement urbain au sein de l'équipe ADES : des recherches portant sur les conflits et les jeux d'acteurs dans les aménagements urbains, et la construction de la nature en ville à partir de travaux sur la réhabilitation de friches urbaines en parcs et en jardins partagés. 